\title{
Los puntos críticos del sistema educativo: el "fracaso escolar" en el análisis de trayectorias en la escuela media de la provincia de Buenos Aires y Ciudad Autónoma de Buenos Aires (2001-2009)*
}

\author{
Tatiana Santillán**
}

\section{Resumen}

¿Qué influencia puede tener el marco institucional sobre las trayectorias escolares? ¿Qué aportes pueden corresponder a partir del análisis de los 'puntos críticos'? En este trabajo analizamos la influencia que tiene la organización institucional del sistema educativo pautado por las leyes de educación en las trayectorias escolares de la escuela media de la provincia de Buenos Aires y Ciudad Autónoma de Buenos Aires (CABA). La metodología que nos permitió observar este impacto fue el seguimiento de 16 cohortes reconstruidas a partir del Relevamiento Anual (RA) que genera la Dirección Nacional de Información y Evaluación de la Calidad Educativa (DINIECE).

\section{Palabras clave}

Trayectorias escolares - trayectorias "teóricas" - trayectorias "reales".

\footnotetext{
* Artículo recibido el 4 de Febrero de 2015. Aceptado el 5 de Junio de 2015.

** Tatiana Santillán es Licenciada en Sociología, Universidad Nacional de Mar del Plata (UNMDP) con la tesis "Análisis de las trayectorias escolares en la escuela media de la provincia de Buenos Aires y Ciudad Autónoma de Buenos Aires (2001-2009)". Actualmente maestranda en Generación y Análisis de Información Estadística. Universidad Nacional de Tres de Febrero (UNTREF).

Correo electrónico: tatianasantillan@hotmail.es
} 


\section{Abstract}

What influence can have the institutional framework on educational tracks? What contributions can correspond to the analysis of the "hot spots"? In this paper we analyze the influence of the institutional organization of the educational system patterned by education law school careers in middle school in the province of Buenos Aires and Ciudad Autónoma de Buenos Aires (CABA). The methodology allowed us to observe this impact was tracking 16 cohorts reconstructed from Relevamiento Anual (RA) provides us with the Dirección Nacional de Información y Evaluación de la Calidad Educativa (DINIECE).

\section{Keywords}

School trajectories - trajectories "theoretical" - trajectories "real".

\section{Introducción}

Desde la creación en el país del sistema de enseñanza media en 1863, esta fue objeto de diversas propuestas de reforma que no alcanzaron consenso para su implementación (DINIECE, 2007: 6). A lo largo del tiempo, se avanzó en pequeñas modificaciones referidas a contenidos (reformas en el currículum) ${ }^{1}$, a metodologías y a la evaluación. Solo en el siglo veinte, en la década de noventa ${ }^{2}$, se realiza la primera reforma educativa en el país. Es interesante advertir que la Ley Federal de Educación de 1993 fue la primera que intentó abarcar todos los niveles educativos -a excepción del nivel superior-, así como la primera en regular la educación secundaria de forma integral (Gallart, 2006) y en la década siguiente se introducen nuevos cambios que quedan plasmados en la Ley Nacional de Educación de 2006.

Las trayectorias de los estudiantes de la escuela media están íntimamente relacionadas con la estructura institucional que plantean las leyes de educación porque son estas las que organizan la estructura teórica de las trayectorias. El concepto 'trayectoria escolar teórica' en el sistema educativo argentino corresponde a la organización secuencial de cuatro niveles de enseñanza: inicial, educación general básica (EGB) y/o primaria, polimodal y/o media y superior universitario y no universitario. El sistema educativo, por lo tanto, estructura la trayectoria teórica, según

\footnotetext{
${ }^{1}$ Para profundizar acerca de las reformas en el currículum en el nivel secundario se recomienda la lectura de la tesis de maestría de Inés Dussel (1997) "Currículum, Humanismo y Democracia en la enseñanza media (1863-1920)".

${ }^{2}$ Los países que también experimentaron en la década pasada reformas en sus leyes de educación son los siguientes: Chile (1990), Guatemala (1991), México (1993), Colombia (1994), Bolivia (1994), El Salvador (1996), Brasil (1996), Republica Dominicana (1997), Paraguay (1998) y Venezuela (1999).
} 
Terigi (2010), a partir de: "la organización del sistema por niveles, la gradualidad del currículum, la anualización de los grados de instrucción y la definición de las edades de inicio y fin de la obligatoriedad escolar" (Terigi, 2010: 3). El concepto de trayectoria escolar tiene como supuesto básico que los recorridos de los sujetos en el sistema siguen la progresión lineal prevista por este en los tiempos marcados por una periodización estándar (Terigi, 2008). Es decir, los recorridos que realizan los sujetos se suponen estandarizados, homogéneos y lineales. Terigi (2007) realiza una distinción entre estas trayectorias escolares denominadas teóricas y las trayectorias escolares 'reales', también denominada por otros autores como "diversidad de trayectorias" (Fainsod, 2006), "jóvenes con trayectorias escolares inconclusas", "trayectorias no encauzadas" (Terigi, 2009) "trayectorias escolares marcadas por la discontinuidad" (Kantor, 2001) o la "intermitencia en las trayectorias escolares" (Kessler, 2004). Estos conceptos remiten a la diferencia que existe entre lo que el sistema educativo estructura idealmente y lo que efectivamente sucede en la práctica.

En este estudio nos preguntamos de qué manera la organización del sistema educativo genera variaciones en las trayectorias escolares de los estudiantes. Observamos cómo la organización institucional pautada por la ley Federal de Educación genera 'puntos críticos' ${ }^{4}$ en las trayectorias de los estudiantes. Nos referimos a 'puntos críticos' en el sistema educativo argentino para marcar las rupturas, las discontinuidades, los quiebres, los alejamientos o distancias que se generan entre las "trayectorias escolares teóricas" y las "trayectorias escolares reales" de los jóvenes de la provincia de Buenos Aires y Ciudad Autónoma de Buenos Aires producidos por la estructura organizacional de las instituciones educativas. Al mismo tiempo, hablamos de "persistencia de puntos críticos" porque pudimos observar, mediante una serie histórica que comienza en el año 2001 y finaliza en el 2009, que los puntos críticos aparecían con la misma frecuencia, comportándose de la misma manera en cada una de las trayectorias de las dieciséis cohortes teóricas que reconstruimos, alertándonos sobre la presencia de estructuras organizacionales educativas que generan problemas en las trayectorias de los estudiantes.

\section{Las trayectorias de los estudiantes de la escuela media y su relación con la estructura institucional que plantean las leyes de educación}

La reorganización del secundario tradicional de cinco años en dos segmentos de tres años (EGB3 y Polimodal) en la provincia de Buenos Aires y la estructura de Ciudad de Buenos Aires donde se mantiene el formato del secundario de cinco años implica

\footnotetext{
3 Flavia Terigi (2009) toma el concepto de "trayectorias no encauzadas" empleado por el European Group for Integrated Social Research (EGRIS) para referirse a las características que presentan las trayectorias de vida de los jóvenes europeos, con referencias al empleo, la escolarización y otros aspectos.

4 Tomamos la idea de persistencia de puntos críticos de Flavia Terigi (2009), quien hace referencia a Almandoz (2000).
} 
empalmes y solapamientos de los datos para la reconstrucción de las trayectorias escolares. Como criterio general y para poder realizar una comparación entre las trayectorias de los estudiantes con dos estructuras organizacionales diferentes, se reconstruyó la serie histórica considerando el nivel medio con una duración de seis años independientemente de la estructura institucional que posea cada distrito. Siguiendo la recomendación de la Dirección Nacional de Información y Evaluación de la Calidad Educativa (DINIECE) y en base a un documento de UNICEF (2011), para mantener la comparabilidad de las estructuras de niveles entre jurisdicciones, se considera como secundaria al tramo del $7^{\circ}$ al $9^{\circ}$ grado de EGB más el nivel polimodal de $1^{\circ}$ a $3^{\circ}$ año para el total de la provincia de Buenos Aires y en Ciudad Autónoma de Buenos Aires se consideró el $7^{\circ}$ grado más el secundario de $1^{\circ}$ a $5^{\circ}$ año.

En las cohortes que estudiamos la reorganización institucional que propone la Ley Nacional de Educación promulgada en el año 2006 no alcanza a tener efectos en las trayectorias escolares de las cohortes reconstruidas ${ }^{5}$. Esto es así porque la ley estipula un periodo de seis años, es decir, hasta el año 2012 para que las escuelas realicen la transición entre la estructura académica que se implementó con la Ley Federal de Educación (1993) y la estructura que plantea la Ley Nacional de Educación (2006).

Las cohortes que estudiamos y que comienzan en el año 2001, en el año que se promulgaba la Ley Nacional de Educación -2006- estaba finalizando su trayectoria; en la cohorte que comienza en el año 2002 faltaban solo dos años para finalizar sus estudios; a la cohorte que comienza en el año 2003 le faltaban tres años para que terminara la trayectoria escolar y a la cohorte que comienza en el año 2004 le faltaban cuatro años para finalizar su trayectoria. En este sentido, las cohortes que comienzan con una organización académica continúan con la misma hasta finalizar sus estudios. Como se puede observar en el cuadro que presentamos a continuación, no tenemos ninguna cohorte que comience a partir de la promulgación de la Ley Nacional de Educación (2006). Con los datos disponibles construimos cuatro (4) cohortes teóricas para cada distrito.

\section{Cuadro 2: Cohortes según año de estudio y año calendario}

\begin{tabular}{|c|l|l|l|l|}
\hline \multirow{2}{*}{$\begin{array}{c}\text { Años de } \\
\text { estudio }\end{array}$} & \multicolumn{3}{|l|}{ Cohortes } \\
\cline { 2 - 5 } & 1 ra & $2 \mathrm{da}$ & 3 era & 4 ta \\
\hline
\end{tabular}

\footnotetext{
${ }^{5}$ Es necesario definir aquí la matrícula escolar como el conjunto de estudiantes de la cohorte, es decir, aquellos en los que se puede observar que cumplen con las trayectorias escolares teóricas esperadas por el sistema educativo y por los reinscriptos. La categoría reinscripto es la que refleja las trayectorias escolares 'reales'. Está compuesta por los repitentes del año anterior y por otras formas de transitar por el sistema educativo, como por ejemplo los ingresos tardíos, los abandonos temporarios, los repitentes de otros años. A estas diversas formas de transitar el sistema educativo lo denominamos bajo el nombre 'otros', ya que no pudimos distinguir, a partir de los datos disponibles, qué situación particular corresponde a cada caso.
} 


\begin{tabular}{|l|l|l|l|l|}
\hline & cohorte & cohorte & cohorte & cohorte \\
\hline 1 & 2001 & 2002 & 2003 & 2004 \\
\hline 2 & 2002 & 2003 & 2004 & 2005 \\
\hline 3 & 2003 & 2004 & 2005 & $\mathbf{2 0 0 6}$ \\
\hline 4 & 2004 & 2005 & $\mathbf{2 0 0 6}$ & 2007 \\
\hline 5 & 2005 & $\mathbf{2 0 0 6}$ & 2007 & 2008 \\
\hline 6 & $\mathbf{2 0 0 6}$ & 2007 & 2008 & 2009 \\
\hline
\end{tabular}

Fuente: Elaboración propia.

Como la estructura organizacional que plantea la Ley Nacional de Educación (2006) no alcanza a tener efectos sobre las cohortes estudiadas necesitamos referirnos a la estructura organizacional que plantea la Ley Federal de Educación (1993). Esto nos indica que debemos analizar los datos de una manera para el total de la provincia de Buenos Aires -incluyendo al Conurbano y al Resto de los Partidos-; y que debemos efectuar otra mirada para analizar a la Ciudad Autónoma de Buenos Aires, ya que al ser diferente la estructura organizacional se enfrenta a diferentes problemas.

\section{3. ¿Fracaso escolar?: los puntos críticos en las trayectorias de las cohortes}

La noción de "fracaso escolar" se ha utilizado para describir y analizar las dificultades en las trayectorias escolares de los estudiantes. Según varios autores (Lus, 1996; Terigi, 2009) este fenómeno es contemporáneo a la escolarización masiva, en el marco de lo que conocemos como "escuela moderna". Donde el "fracaso escolar" fue interpretado desde el comienzo como un modelo patológico individual y el fracaso masivo fue caracterizado como la suma de los fracasos individuales. Terigi (2009) sostiene que el "fracaso escolar", desde esta perspectiva, convierte en problema de los estudiantes lo que en verdad son los límites de la estructura organizacional del sistema educativo. La mirada que culpabiliza al estudiante ha recibido importantes críticas que surgen de la pregunta: ¿Quién es el que fracasa? ¿Es el estudiante o la organización institucional del sistema educativo?

Antes de entrar de lleno en el análisis de datos, dada la especificidad de las estadísticas educativas, es necesario precisar algunas definiciones: la matrícula escolar se compone por los estudiantes de la cohorte, es decir aquellos en los que se puede observar que cumplen con las trayectorias escolares teóricas esperadas por el sistema educativo y por los reinscriptos. La categoría reinscripto es la que refleja las trayectorias escolares 'reales'. Está compuesta por los repitentes del año anterior y por otras formas de transitar por el sistema educativo, como por ejemplo, los ingresos tardíos, los abandonos temporarios, los repitentes de otros años. A estas diversas formas de transitar el sistema educativo lo denominamos bajo el nombre 'otros', ya que no pudimos distinguir, a partir de los datos disponibles, qué situación particular corresponde a cada caso. 
Los datos que analizamos en este trabajo dan cuenta de que, independientemente del año calendario en que los estudiantes se encuentren cursando, se observan puntos críticos regulares en el tiempo que corresponden con la organización institucional de la escuela. En las trayectorias escolares de las cohortes del total de la provincia de Buenos Aires, Conurbano y Resto de los Partidos observamos la persistencia de dos puntos críticos y en las trayectorias de las cohortes escolares de la Ciudad de Buenos Aires observamos un punto crítico que se repite en la trayectoria de cada una de las cohortes.

\section{1 Los puntos críticos en provincia de Buenos Aires}

- En la provincia de Buenos Aires, Conurbano y Resto de los Partidos observamos que en el paso del tercer al cuarto grado/año de estudio la proporción de los reinscriptos en relación con la matrícula total aumenta considerablemente. Al mismo tiempo disminuye la proporción de los estudiantes de la cohorte en relación con la matrícula total. En el paso de cuarto a quinto grado/año de estudio observamos que disminuye el porcentaje de la matrícula de los reinscriptos respecto al año anterior, pero si lo comparamos con el tercer grado/año de estudio este valor continúa siendo alto.

Gráfico 1: Puntos críticos Buenos Aires, Conurbano y Resto de los Partidos. Porcentaje de la cohorte sobre el resto de la matrícula en cada grado/año de estudio. 


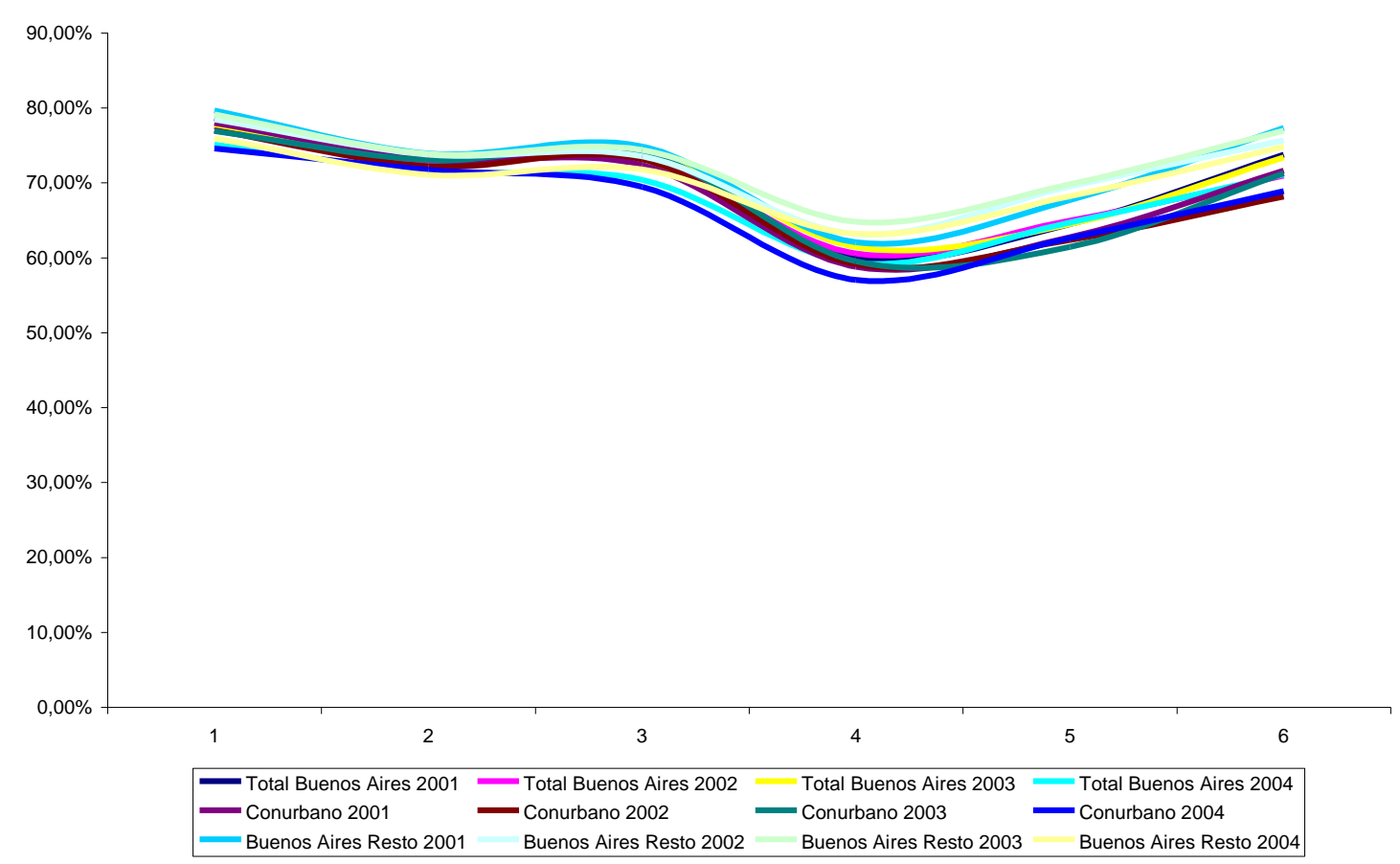

Fuente: Elaboración propia en base a los datos del DINIECE.

Para realizar el gráfico 1 utilizamos el porcentaje de la matrícula de las cuatro cohortes correspondientes al año 2001, 2002, 2003 y 2004 en cada año/grado de estudio, es decir durante la trayectoria que realizó cada una de las cohortes en los distritos: total de la provincia de Buenos Aires, Conurbano y Resto de los Partidos. En este gráfico combinamos las trayectorias de 12 cohortes y lo que podemos observar es que todas ellas se comportan de la misma manera con variaciones que responden a la cantidad de la matrícula inicial de cada una de las cohortes. Al mismo tiempo, podemos observar claramente que los puntos críticos que señalábamos corresponden al paso del tercero a cuarto año de estudio, en este caso, corresponde al paso de nivel EGB3 al nivel Polimodal.

\subsection{El punto crítico de Ciudad Autónoma de Buenos Aires}

- En la Ciudad Autónoma de Buenos Aires observamos un punto crítico en la trayectoria de cada una de las cohortes. En el paso del primer al segundo grado/año de estudio la proporción de los reinscriptos en relación con la matrícula total aumenta al doble. Aquí se observa también que el problema está en el paso de nivel, de primario a secundario.

Gráfico 2: Puntos críticos de Ciudad Autónoma de Buenos Aires. Porcentaje de la cohorte sobre el resto de la matrícula en cada grado/año de estudio. 


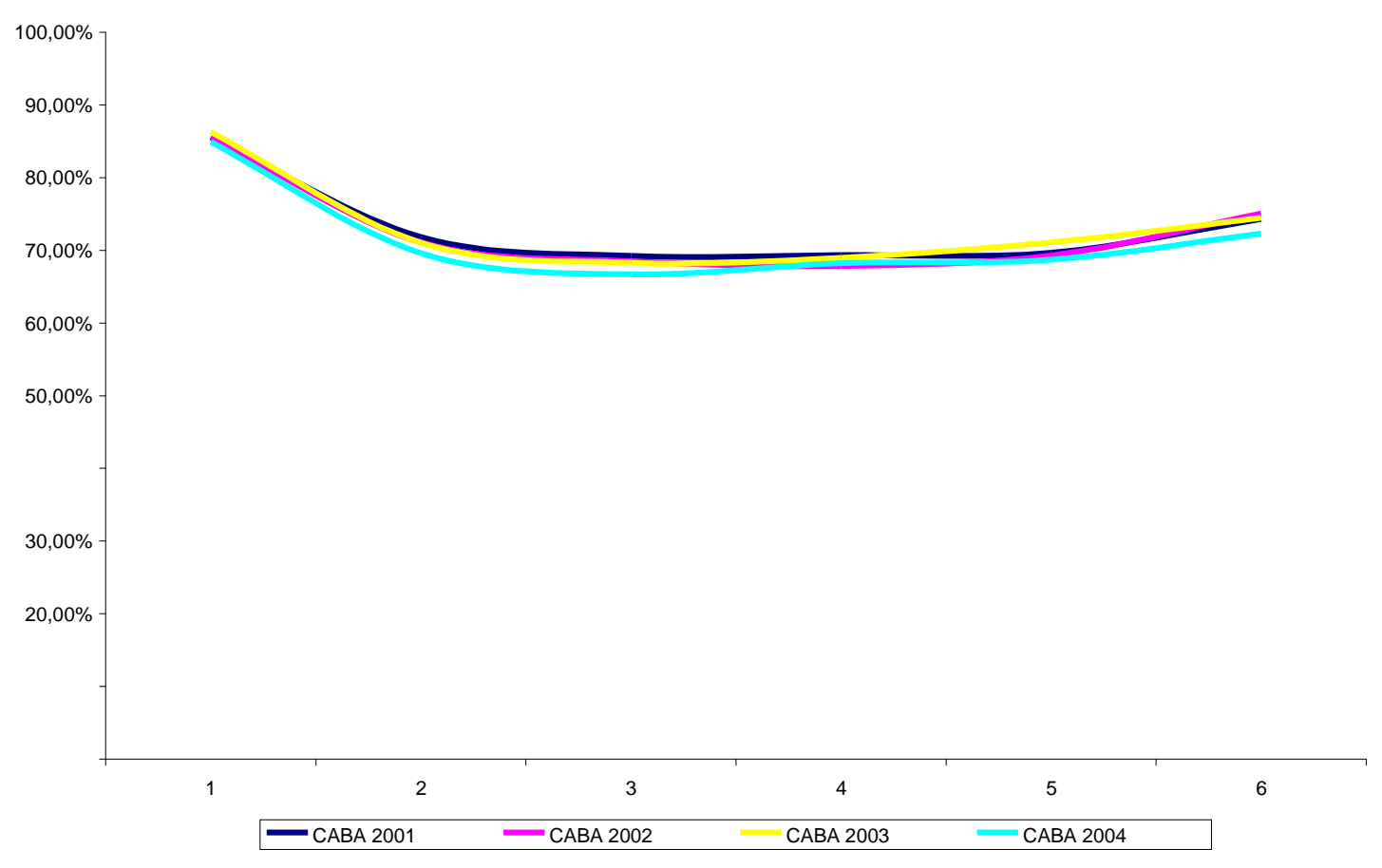

Fuente: Elaboración propia en base a los datos del DINIECE.

Para realizar el gráfico 2 utilizamos los datos de Ciudad Autónoma de Buenos Aires que corresponden a cada una de las cohortes (2001, 2002, 2003 y 2004) a lo largo de su trayectoria, es decir por cada grado/año de estudio. Esta decisión se basó en que los puntos críticos del total de la provincia de Buenos Aires (en conjunto con el Conurbano y con el Resto de los Partidos) son distintos a los de Ciudad Autónoma de Buenos Aires. En Ciudad Autónoma de Buenos Aires se observa que las cuatro trayectorias de cada una de las cohortes se comportan de la misma manera y al mismo tiempo nos permite mostrar el punto crítico que señalamos anteriormente en el paso de primero a segundo grado/año. El punto crítico se observa en el cambio de nivel, es decir en el paso del primario al secundario. En este sentido, Terigi (2009) sostiene que en términos de trayectorias escolares "muchos de quienes egresan de la escuela primaria o sus equivalentes no se incorporan al nivel secundario. En términos generales, las transiciones entre niveles son puntos críticos de las trayectorias reales de los alumnos en nuestros sistemas" (Terigi, 2009: 23).

El gráfico 3, que presentamos a continuación, intenta mostrar las diferencias en los puntos críticos entre Buenos Aires, Conurbano, Resto de los Partidos y Ciudad Autónoma de Buenos Aires. Se puede observar claramente la persistencia de los dos puntos críticos en la provincia de Buenos Aires (conjuntamente con Conurbano y Resto de los Partidos) que mencionábamos anteriormente. Recordemos que esta situación se generaba en el paso de tercero a cuarto grado/año de estudio en las trayectorias de los estudiantes y que persistía también en el paso de cuarto a quinto grado/año de estudio. En Ciudad Autónoma de Buenos Aires se observa que el punto crítico en la 
trayectoria de los estudiantes ocurre en el paso de primero a segundo año/grado de estudio.

Gráfico 3: Puntos críticos Buenos Aires, Conurbano, Resto de los Partidos y Ciudad Autónoma de Buenos Aires. Porcentaje de la cohorte sobre el resto de la matrícula en cada grado/año de estudio.

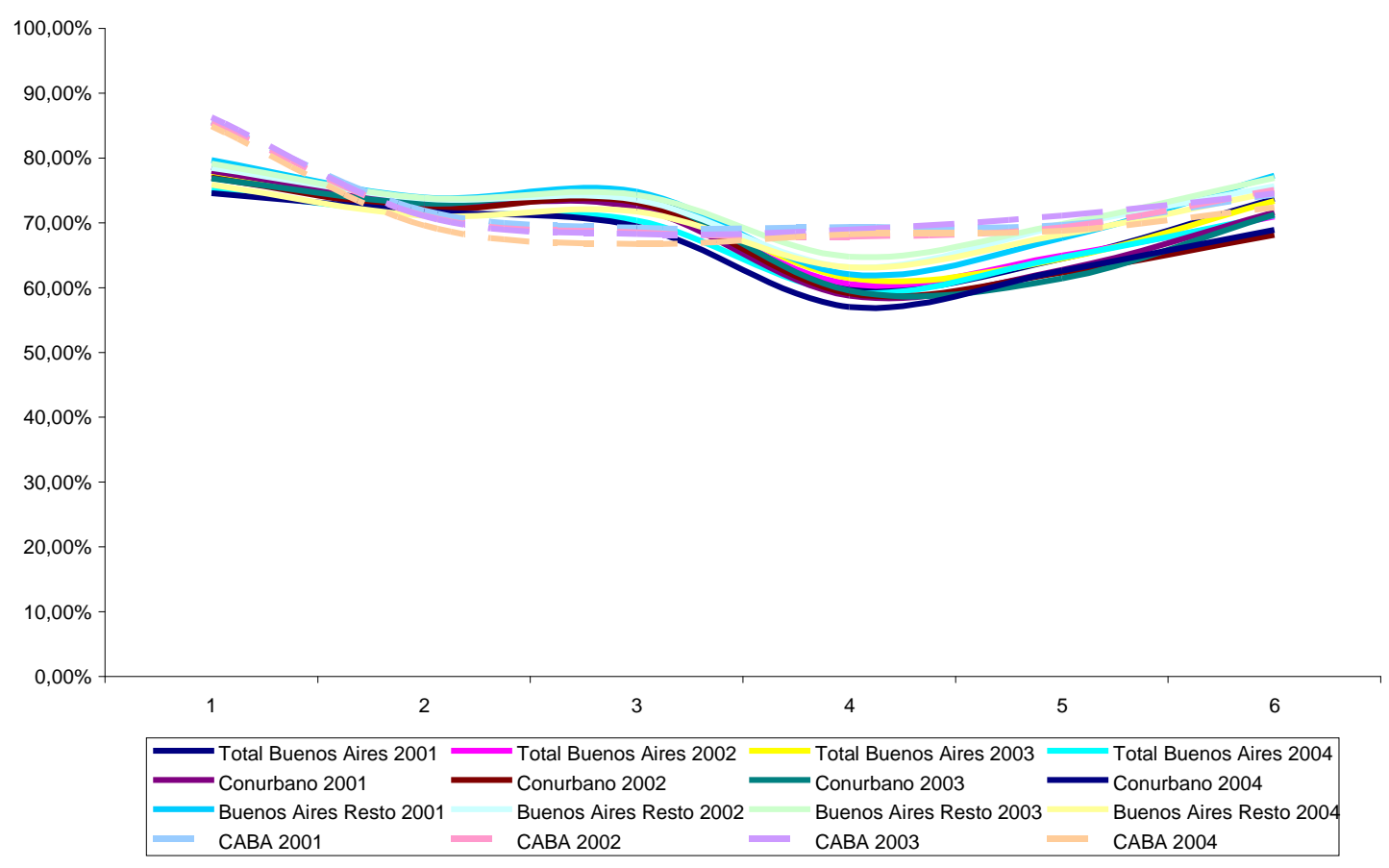

Fuente: Elaboración propia en base a los datos del DINIECE.

Es importante remarcar que, aunque los puntos críticos en ambos distritos (Buenos Aires en su conjunto y Ciudad Autónoma de Buenos Aires) ocurran en diferentes momentos de la trayectoria escolar, esto se debe a la organización institucional del sistema educativo, ya que en ambos distritos es diferente. Lo interesante es que aunque estas situaciones críticas se den en momentos cronológicos diferentes ocurren siempre en el paso del nivel primario al secundario o polimodal dependiendo del caso.

En sintonía con lo anterior, realizamos el gráfico 4, de lectura más simple. Este gráfico lo realizamos con el promedio de la matrícula de las cuatro cohortes correspondientes a los años 2001, 2002, 2003 y 2004 de total Buenos Aires, Conurbano y Resto de los Partidos a lo largo de la trayectoria, es decir por año/grado de estudio y luego realizamos el promedio del promedio que habíamos obtenido anteriormente. El mismo procedimiento empleamos para Ciudad Autónoma de Buenos Aires, pero en este caso, al ser un solo distrito, solo sacamos el promedio de la matrícula de las cuatro cohortes a lo largo de la trayectoria, es decir, por año/grado de estudio.

Este gráfico intenta mostrar los puntos críticos de las trayectorias escolares de los estudiantes del total de la provincia de Buenos Aires (en conjunto con Conurbano y 
Resto de los Partidos) y hacer aún más visible la diferencia que hay en las trayectorias escolares de los estudiantes de Ciudad Autónoma de Buenos Aires.

\section{Gráfico 4: Puntos críticos Buenos Aires, Conurbano, Resto de los Partidos y Ciudad Autónoma de Buenos Aires. Porcentaje de la cohorte sobre el resto de la matrícula en cada grado/año de estudio.}

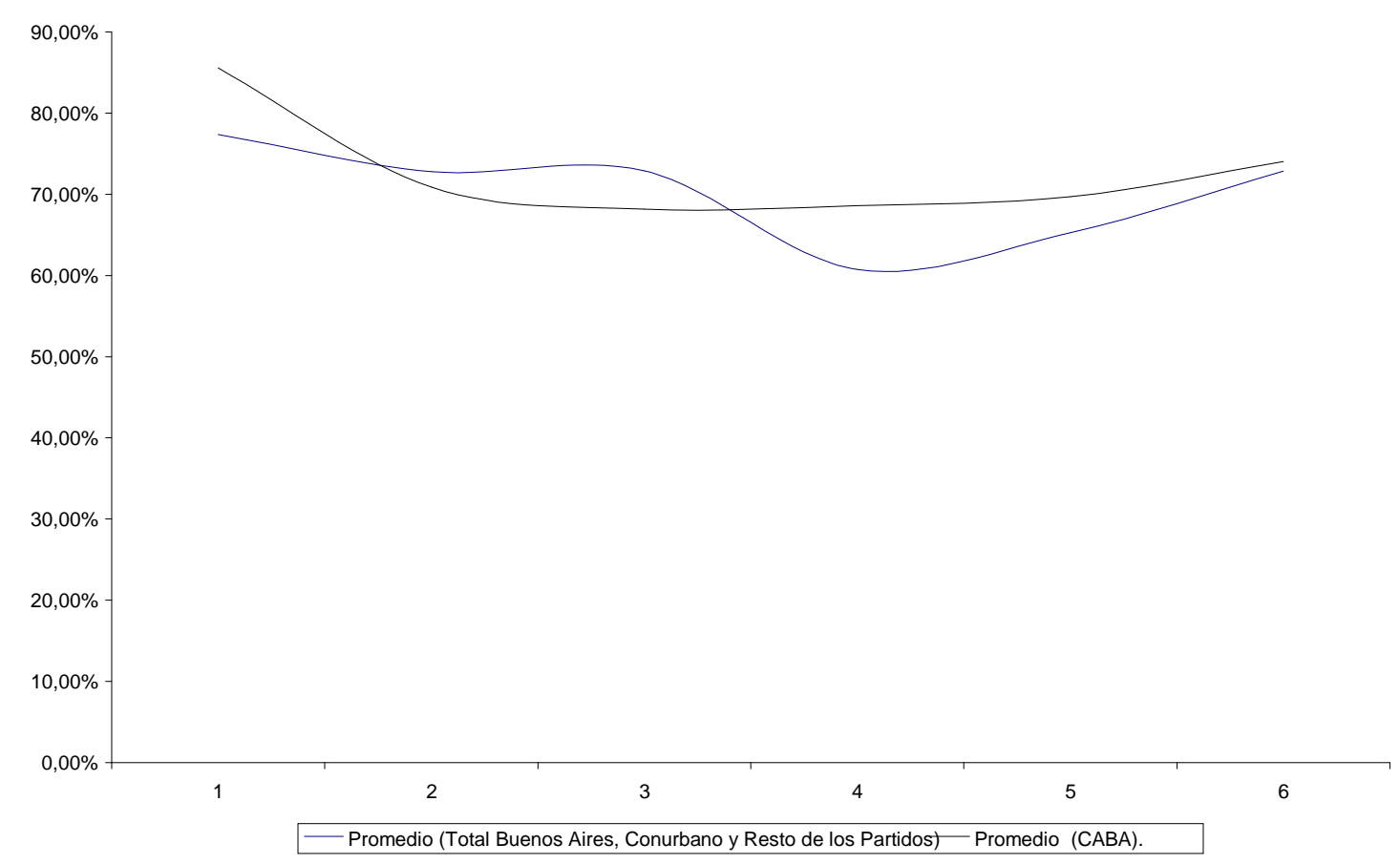

Fuente: Elaboración propia en base a los datos del DINIECE.

\section{La huella de las trayectorias reales en las trayectorias teóricas}

Al reconstruir las trayectorias en las cohortes correspondientes al año 2001 no pudimos desagregar la categoría reinscriptos ${ }^{6}$ en "repitentes" del año anterior" y "otros" ${ }^{8}$ ya que no disponíamos de los datos correspondientes al año 2000, para ninguno de los distritos analizados: total Buenos Aires, Conurbano, Buenos Aires Resto y Ciudad Autónoma de Buenos Aires. Para todo el periodo analizado la proporción de los "repitentes del año anterior", respecto a la categoría "otros", es menor a la mitad. El

\footnotetext{
${ }^{6}$ Según glosario de DINIECE (2003) alumno que registra en un año de estudio en el que ya se había inscripto en algún ciclo lectivo anterior, sin completar la cursada, es decir, que no alcanzó a integrar el universo de evaluación o acreditación de aprendizajes (matrícula final).

${ }^{7}$ Según glosario de DINIECE (2003) alumno que cursa por segunda vez o más veces el mismo año de estudio o ciclo, por no haberlo promovido en su anterior oportunidad.

8 Recordemos la definición de la categoría "otros" que engloba diferentes formas de transitar por el sistema educativo, como por ejemplo los ingresos tardíos, los abandonos temporarios, los repitentes de otros años.
} 
porcentaje de repitentes para el último grado/año de estudio es el menor porcentaje registrado durante todo el periodo para cada una de las cohortes. Para todas las cohortes analizadas la composición de la categoría "otros" en relación con los reinscriptos alcanza valores de más del doble en comparación con los "repitentes del año anterior" durante toda las trayectorias de las cohortes.

La categoría "otros" es entonces muy significativa en la composición de los reinscriptos. Sin embargo, nos encontramos con un problema, ya que esta categoría a partir de los datos disponibles no podemos conocer a qué situación particular corresponde en cada caso. Es decir, no sabemos qué proporción de los inscriptos habían abandonado sus estudios previamente y qué proporción eran estudiantes que repitieron más de una vez o ingresaron tardíamente a la escuela; tampoco pudimos saber en qué grado/año de estudio repitieron. Al desconocer las proporciones de la composición de esta categoría es difícil que se generen políticas educativas efectivas que atiendan a cada uno de los casos particulares, ya que estas deben atender a problemáticas distintas. De este modo, se desconocen las dimensiones del problema y los subgrupos que se podrían identificar. No es lo mismo generar políticas educativas ante el problema de la repitencia que ante el problema del abandono. Aunque se sabe que en muchos casos la antesala del abandono es la repetición reiterada (Mancebo: 2011). Por otro lado, dejar de asistir al nivel secundario no implica necesariamente el abandono escolar. Muchas veces los jóvenes dejan de asistir a la escuela temporalmente pero luego se reintegran a su curso; y por otro lado a veces un estudiante tiene un número muy elevado de inasistencias a lo largo del año lectivo pero en ningún momento interrumpe su concurrencia (Mancebo: 2011). Cuando hablamos de abandono ise trata del alumno que deja de ir a la escuela en la que estaba matriculado o a la escuela en general? ¿Ha dejado esa escuela y se ha matriculado en otra? ¿Es el estudiante que se ha mudado de localidad? ¿Es el extranjero que deja de asistir a la escuela porque vuelve a su país?

Las autoras Cappellacci y Miranda (2007) sostienen la hipótesis de que a partir del año 2003, las mayores posibilidades ocupacionales podrían estar compitiendo con la escolarización de los jóvenes; en este sentido, una significativa proporción de ellos habría optado por continuar sus estudios en el espacio de la educación de adultos. Las autoras comparan la evolución de los jóvenes que asisten al nivel medio entre los años 2002 y 2006 en función del tipo de oferta -educación común o educación de adultos- y arriban a la conclusión de que "un poco más del $70 \%$ de la disminución de la matrícula de la educación común podría explicarse por el incremento de jóvenes en la educación de adultos". (Cappellacci y Miranda, 2007: 10). Otro estudio que afirma las hipótesis que plantean Cappellacci y Miranda (2007) es el que fue realizado posteriormente por Filmus y Carcar (2010) que da cuenta de este fenómeno: a partir del año 2003 en donde el país empieza a recuperarse económicamente, los jóvenes tienen mayores posibilidades de acceder a un empleo y "tienden en mayor proporción a optar por 
concurrir a las escuelas secundarias de adultos, en su mayoría nocturnas" (Filmus y Carcar, 2010: 130). Esto ocurre porque las escuelas secundarias para adultos son más flexibles y permite a los jóvenes combinar el estudio con el trabajo. Los autores sostienen que aproximadamente la mitad de los jóvenes de entre 15 a 19 años que ingresaron al mercado laboral se dedicaron a combinar el estudio con el trabajo y la otra mitad solo se dedicó a trabajar. Solo muy pocos, en este rango etario, no estudian ni trabajan.

\section{Consideraciones finales}

El sistema educativo argentino está pensado con una organización institucional que pauta las "trayectorias teóricas" de los estudiantes. Observamos que las trayectorias de los estudiantes de los distritos analizados no siempre responden a estas "trayectorias teóricas" esperadas, sino que estas están signadas por diversas situaciones, como por ejemplo: los ingresos tardíos, los abandonos temporarios, los repitentes -entre los que podemos distinguir "repitentes del año anterior" y "repitentes de otros años"-, todas estas posibilidades se engloban en el concepto de "reinscriptos" y son generados no solo por la organización propia del sistema educativo, sino también por factores exógenos. En este sentido, las diferencias entre las trayectorias teóricas y reales 'hablan' de las dificultades por las que atraviesan los jóvenes para transitar por el sistema educativo.

En este estudio nos dedicamos a observar cómo la organización institucional pautada por las reformas educativas genera 'puntos críticos' en el sistema educativo. Nos interrogamos sobre de qué manera la organización propia del sistema educativo genera variaciones en las trayectorias escolares de los estudiantes.

Los puntos críticos en el sistema educativo son producto del marco institucional que organiza las trayectorias de los estudiantes y se relacionan con objetivos y problemas de largo alcance, a saber:

En primer lugar, la relación entre escolarización y entrada al mundo del trabajo. Recordemos que con la estructura que planteaba la Ley Federal de Educación (1993) en el tercer año de estudio se estaría finalizando la escolarización obligatoria -que alcanzaba a los 14-15 años de edad. Aquí, para muchos estudiantes se generaba un dilema entre seguir estudiando o trabajar, ya que a un adolescente se lo consideraba como parte de la población económicamente activa a partir de los 13 años de edad ${ }^{9}$.

En segundo lugar, el paso del nivel primario al secundario. Muchos estudiantes culminan con éxito el ciclo escolar y se ven obligados a cambiar de institución al matricularse, ya que muchas escuelas solo tenían hasta la EGB3. Esta situación genera, en muchos casos, que el estudiante interrumpa sus estudios. Un estudio del DINIECE en

\footnotetext{
${ }^{9}$ En el año 2008 se modificaron las leyes No 20 774; 22 248; 23 551; 25013 y el decreto de ley No 326/56 que prohíbe el trabajo infantil y protege el trabajo adolescente. Fija la edad mínima de admisión en un empleo en 16 años, quedando prohibido el trabajo de menores de 16 años.
} 
conjunto con UNICEF (2004) señala que faltan establecimientos educativos, especialmente en el caso del tercer ciclo de la EGB y el nivel Polimodal. En este trabajo pudimos dar cuenta de que las variaciones en las trayectorias escolares de los estudiantes se dan con más notoriedad en el paso del nivel primario al secundario, (como Ciudad Autónoma de Buenos Aires), o en el paso del nivel EGB3 al polimodal, (como en el total de la provincia de Buenos Aires, Conurbano y Resto de los Partidos). El pasaje de un ciclo a otro representa fuertes dificultades de adaptación para los niños que ingresan a instituciones en las cuales hay "criterios difusos" (DINIECE-UNICEF, 2004) en relación con el sentido de la actividad escolar. Al mismo tiempo, Maddoni y Sipes (2010) sostienen que "dentro del trayecto que recorren los niños, los pasajes entre niveles son cruciales y es donde se producen las mayores repeticiones y abandonos" (Maddoni y Sipes, 2010: 54). Esta situación también la ha registrado Rossano (2006) quien ha estudiado el pasaje del nivel primario al secundario. La autora sostiene que en esta transición los estudiantes pierden visibilidad en el sistema educativo, ya que "nadie los espera ni los busca pues ya han egresado de la escuela primaria pero todavía no pertenecen a ninguna otra institución educativa" (Rossano, 2006: 300, citado en: Terigi, 2009: 40).

A la luz de los cambios organizacionales introducidos en la Ley Nacional de Educación (2006), nos preguntamos: ¿Los cambios institucionales plasmados en la Ley Nacional de Educación (2006) generarán transformaciones en las trayectorias escolares de los estudiantes? ¿Se puede pensar que las trayectorias que analizamos van a ser similares luego de la implementación de la ley? ¿Cómo se pensó el problema del paso del nivel primario al secundario? ¿La Ley Nacional de Educación (2006) generará cambios en este sentido?

\section{Bibliografía}

\section{Documentos Oficiales}

Ley Federal de Educación N. ${ }^{\text {ro }} 24$ 195. (1993). Ministerio de Cultura y Educación. Disponible en: [http://www.bnm.mcye.gov.ar/demo2/documen/leyes/leyf/leyf.htm] (Última consulta: Octubre de 2012).

Ley de Educación Nacional N. ${ }^{\text {ro }} 26$ 206. (2006). Ministerio de Cultura y Educación. Disponible en: [http://www.me.gov.ar/doc_pdf/ley_de_educ_nac.pdf] (Última consulta: Octubre de 2012)

DINIECE-UNICEF. (2004). Las dificultades en las trayectorias escolares de los alumnos. Un estudio en las escuelas de nuestro país. Disponible en: [http://diniece.me.gov.ar/images/stories/diniece/investigacion_programas/investigac iones/trayescolar.pdf] (Última consulta: Marzo de 2013). 


\section{Bibliografía citada}

BINSTOCK, Georgina y CERRUTTI, Marcela. (2005). Carreras truncadas: el abandono escolar en el nivel medio en la Argentina. Buenos Aires: UNICEF.

BRISCIOLI, Bárbara. (2009). Tensiones que plantea la extensión de la obligatoriedad de la educación secundaria. Ponencia presentada en: Congreso Internacional de Filosofía y Educación en Nuestra América. Políticas, Escuelas e Infancias. ISBN: 978-987-9441-38-1

CAPELLACCI, Inés y MIRANDA, Ana. (2007). La obligatoriedad de la educación secundaria en Argentina. Deudas pendientes y nuevos desafíos. Serie la educación en debate. Documentos de la DINIECE $N^{\circ} 4$. Disponible en: [http://repositorio.educacion.gov.ar/dspace/bitstream/handle/123456789/96787/ EL000990.pdf?sequence=1] (Última consulta: Octubre de 2012).

CARCAR, Fabiola y FILMUS, Daniel. (2010). Educación y trabajo en América Latina. Y Argentina en las dos últimas décadas. En: FILMUS, Daniel (Comp.) Crisis, transformación y crecimiento: América Latina (2000-20010). Buenos Aires: Eudeba.

DIKER, Gabriela y FEENEY, Silvina. (1998). La evaluación de la calidad. Un análisis del discurso oficial. En: Revista del Instituto de Investigaciones en Ciencias de la Educación. Año VII, No 12. Agosto de 1998.

DUSSEL, Inés. (1997). Currículum, humanismo y democracia en la enseñanza media. (1863-1920). Buenos Aires: Universidad de Buenos Aires y Facultad Latinoamericana de Ciencias Sociales (FLACSO).

FAINSOD, Paula. (2006). Embarazo y maternidad adolescente en la escuela media. Buenos Aires: Miño y Dávila.

GALLART, María Antonia. (2006). La construcción social de la escuela media: una Aproximación institucional. Buenos Aires: La Crujía.

KESSLER, Gabriel. (2004). Sociología del delito amateur. Buenos Aires: Paidós.

LUS, María Angélica. (1996). De la integración escolar a la escuela integradora. Buenos Aires: Paidós.

MADDONI, Patricia et. al. (2010). El trabajo del director y el cuidado de las trayectorias educativas. Buenos Aires: Ministerio de educación de la Nación. [Disponible en: http://repositorio.educacion.gov.ar] (Última consulta: Diciembre de 2012).

MANCEBO, María Ester et. al. (2011). La exclusión educativa en los países del Cono Sur. Ponencia presentada en: $X$ Jornadas de Investigación de la Facultad de Ciencias Sociales, Universidad de la República de Montevideo.

MARCHESI, Álvaro. (2003). El fracaso escolar en España. Documento de trabajo. Madrid: Fundación alternativa. Disponible en: [http://es.scribd.com/doc/97436510/FracasOes-Col-Are-Span-A] (Última consulta: Diciembre de 2012).

TERIGI, Flavia. (2007). Los desafíos que plantean las trayectorias escolares. Paper presentado en el III Foro Latinoamericano de Educación Jóvenes y docentes. La escuela secundaria en el mundo de hoy. Organizado por la Fundación Santillana. 
Buenos Aires, 28, 29 y 30 de mayo de 2007. Disponible en: [www.ieo.edu.ar/promedu/trayescolar/desafios.pdf] (Última consulta: abril de 2012).

TERIGI, Flavia. (2008). Detrás, está la gente. En: Organización de Estados Americanos Proyecto Hemisférico. Elaboración de Políticas y Estrategias para la Prevención del Fracaso Escolar. Seminario Virtual de Formación. Lecturas acerca de las políticas educativas de inclusión e igualdad. Disponible en: [http://www.porlainclusionmercosur.educ.ar/seminario2008/clase1_titulo1.html] (Última consulta: Febrero de 2013).

TERIGI, Flavia. (2009). El fracaso escolar desde la perspectiva psicoeducativa: hacia una reconceptualización situacional. En: Revista Iberoamericana de Educación № 50. pp. 23-39.

TERIGI, Flavia. (2010). La asistencia escolar en la actualidad. Trayectorias educativas en 8 países de América Latina. En: En la perspectiva de las trayectorias escolares. Sistema de Información de Tendencias Educativas en América Latina (SITEAL). Disponible en: [http://atlas.siteal.org/node/7/lecturas_posibles] (Última consulta: Febrero de 2013). 